\title{
ID number: 60070: The Development of Cavity Ringdown Spectroscopy as a Sensitive Continuous Emission Monitor for Metals
}

July 14, 2000

PI: George P. Miller Mississippi State University, 205 Research Boulevard, Starkville MS 39759 (662) 3257631 email: miller@ dial.msstate.edu

\section{Co-PI: Christopher B. Winstead, Mississippi State University, 205 Research Boulevard, Starkville MS 39759}

\section{Graduate Students: 1}

\section{Research Objective:}

The aim of this study is to evaluate cavity ringdown spectroscopy (CRDS) as an ultrasensitive technique for trace analysis of metals. Potential applications of CRDS to meet stated Department of Energy needs include:

Mercury Continuous Emission Monitor

Multi-Metal Emissions Monitor

Radionuclide Detector and Monitor

A full description of the technique can be found in Ref. 1. Briefly, CRDS is based upon the measurement of the rate of light absorption in a closed optical cavity.

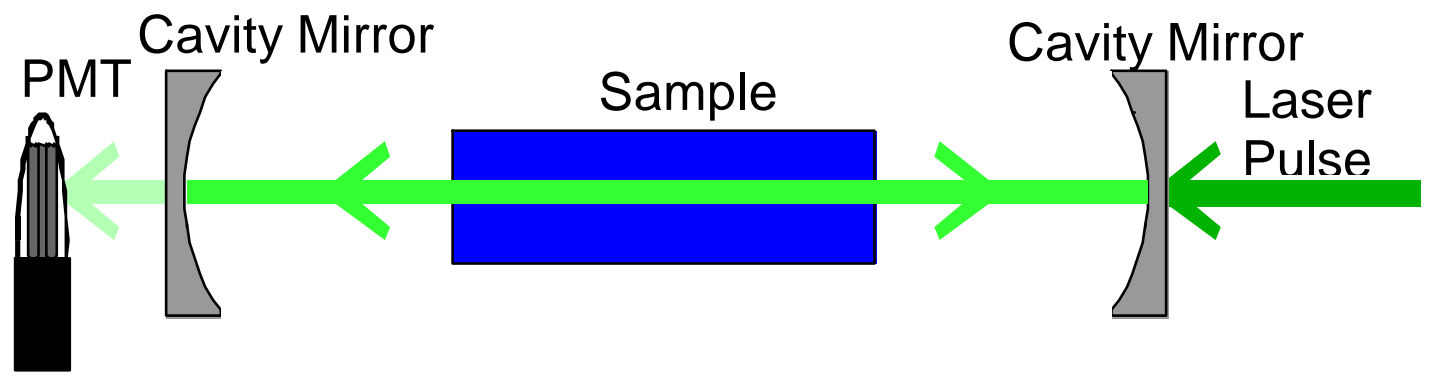

A laser pulse is injected into a stable optical cavity through one of the cavity mirrors. This light pulse is trapped between the mirror surfaces and decays exponentially over time at a rate determined by the round trip losses within the cavity. When used for trace analysis, the primary loss mechanisms governing the decay time are mirror reflectivity losses, atomic absorption from the sample, and Rayleigh scattering from air in the cavity. The decay time is given by

$$
\tau=\frac{d}{c\left[(1-R)+\alpha l_{s}+\beta d\right]}
$$

where $\mathrm{d}$ is the cavity length, $\mathrm{R}$ is the reflectivity of the cavity mirrors, $\alpha$ is the familiar Beer's Law absorption coefficient of a sample in the cavity, $1_{S}$ is the length of the optical path through the sample (i.e., approximately the graphite furnace length), $\beta$ is the wavelength-dependent Rayleigh scattering attenuation coefficient, and $\mathrm{c}$ is the speed of light. Thus, variations in $\alpha$ caused by changes in the sample concentration are reflected in the ringdown time. As the sample concentration increases (i.e., $\alpha$ increases), the ringdown time decreases yielding an absolute measurement for $\alpha$. With the use of suitable mirrors, it is possible to achieve thousands of passes through the sample. This results in an effective path length reaching into the kilometers and a corresponding increase in sensitivity. An additional benefit is that it is not subject to collisional 
quenching and the branching that occur in techniques such as laser-excited atomic fluorescence (LEAFS).

\section{Research Progress and Implications:}

This report summarizes our progress and achievements 30 months into a 3-year project to evaluate the potential of cavity ringdown spectroscopy as a sensitive, continuous emission monitor for toxic metals. Considerable effort has gone into evaluating various atomization sources, the follow sections highlight the results obtained up to this point.

\section{Highlights}

\section{Cold Vapor Mercury Cavity Ringdown Spectrometry:}

1. First published paper on Cold Vapor Hg-Cavity Ringdown Spectroscopy (ref. 2).

2. Using an absorption cell 0.18 meters in length, detection limits of 0.027 and $0.12 \mathrm{ng}$ were obtained using peak area and peak height measurements respectively. For the peak area measurement, this corresponds to a gas phase concentration of less than 25 $\underline{\mathrm{ng} / \mathrm{m}^{3}}$.

(For comparison, using a similar absorption cell, standard AAS yielded an $\mathrm{Hg}$ detection limit (peak height) of $9 \mathrm{ng}$, (gas phase $\sim 830 \mathrm{ng} / \mathrm{m}^{3}$ ).

\section{Electrothermal-CRDS}

3. First published paper on Electrothermal Atomization-Cavity Ringdown Spectroscopy (ref. 3).

2. Non-optimized GF-CRDS detection limits obtained for $\mathrm{Hg}$ and $\mathrm{Pb}$ are better than can be achieved by commercial GF-AAS instruments.

3. Results established the viability of the technique and indicate significant potential. However, as this is a transient technique $(<1 \mathrm{~s})$, the low repetition rate $(20$ $\mathrm{Hz}$ ) of our present dye laser system prevents a full evaluation at this point. Results were highlighted in Analytical Chemistry -News 71, 660A 1999.

\section{ICP-CRDS}

1. First published paper on cavity ringdown measurements in an argon ICP (Ref.4).

2. Detection sensitivity shown to approach theoretical limits.

3. ICP-CRDS stability improved from typically a few percent variation in baseline ringdown times to better than $1 \%$.

4. First cavity ringdown measurements in an air-ICP.

5. Comparison of measured isotopic abundance of $\mathrm{Hg}$ (in an Air-ICP) with the theoretical estimates.

These results clearly demonstrate both the viability and sensitivity of analytical atomic cavity ringdown spectroscopy. The most immediate implication of this research to DOE/EM is in the development of a $\mathrm{Hg}$ continuous emission monitor. We have been shown that required sensitivity can be easily reached without the pre-concentration, and purging with argon, required by other proposed methods.

\section{Planned Activities}

In the remaining period of the grant, work will focus on completing the present research, including the determination of isotopic abundance using cavity ringdown spectroscopy. Two papers; one giving a detailed comparison of coupling three different atomization sources with cavity ringdown for the detection of mercury, the other, a detailed study of ICP-cavity ringdown spectroscopy will be completed and submitted for publication. An Invited presentation of the results obtained under this grant will be presented at the FACSS Conference September 2000 in Nashville.

\section{Information Access}


1. G. P. Miller and C. B. Winstead. Cavity Ringdown Laser Absorption Spectroscopy. Encyclopedia of Analytical Chemistry, Editor R. A. Meyers. Wiley \& Sons Oct 2000.

2. S. Tao, F. Mazzotti, C. B. Winstead and G. P. Miller. Determination of elemental mercury by cavity ringdown spectroscopy. Analyst, 125, (6) 1021 (2000).

3. C. B. Winstead, F. J. Mazzotti, J. Mierzwa, and G. P. Miller. Preliminary results for electrothermal atomization-cavity ringdown spectroscopy (ETA-CRDS. Anal. Commun., 36, 277 (1999). [Highlighted in Analytical Chemistry 71, 660A (1999).]

4. G. P. Miller and C. B. Winstead. Inductively Coupled Plasma Cavity Ringdown Spectrometry. J. Anal. Atom. Spectro. 12, 907 (1997).

5. F. Mazzotti, S. Tao, C. Wang, C. B. Winstead and G. P. Miller. Analytical Atomic Cavity Ringdown Spectroscopy - A Comparison of Three Atomization Sources. In preparation.

6. C. Wang, F. Mazzotti, C. B. Winstead and G. P. Miller. A detailed study of ICP-Cavity Ringdown Spectroscopy. In preparation. 